\title{
On $n$-Tupled Coincidence Point Results in Metric Spaces
}

\author{
Mohammad Imdad, ${ }^{1}$ Ahmed H. Soliman, ${ }^{2,3}$ Binayak S. Choudhury, ${ }^{4}$ and Pradyut Das ${ }^{4}$ \\ ${ }^{1}$ Department of Mathematics, Aligarh Muslim University, Aligarh 202 002, India \\ ${ }^{2}$ Department of Mathematics, Faculty of Science, Al-Azhar University, Assiut 71524, Egypt \\ ${ }^{3}$ Department of Mathematics, Faculty of Science, King Khalid University, Abha 9004, Saudi Arabia \\ ${ }^{4}$ Department of Mathematics, Bengal Engineering and Science University, Shibpur, Howrah, West Bengal 711103, India
}

Correspondence should be addressed to Mohammad Imdad; mhimdad@yahoo.co.in

Received 12 November 2012; Revised 1 February 2013; Accepted 5 February 2013

Academic Editor: Qingkai Kong

Copyright ( 2013 Mohammad Imdad et al. This is an open access article distributed under the Creative Commons Attribution License, which permits unrestricted use, distribution, and reproduction in any medium, provided the original work is properly cited.

We prove some $n$-tupled coincidence point results whenever $n$ is even. We give here several new definitions like $n$-tupled fixed point, $n$-tupled coincidence point, and so forth. The main result is supported with the aid of an illustrative example.

\section{Introduction and Preliminaries}

The classical Banach Contraction Principle proved in complete metric spaces continues to be an indispensable and effective tool in theory as well as applications which guarantees the existence and uniqueness of fixed points of contraction self-mappings besides offering a constructive procedure to compute the fixed point of the underlying map. There already exists an extensive literature on this topic, but keeping in view the relevance of this paper, we merely refer to [1-14].

In 2006, Gnana Bhaskar and Lakshmikantham initiated the idea of coupled fixed point in partially ordered metric spaces and proved some interesting coupled fixed point theorems for mapping satisfying a mixed monotone property. In recent years, many authors obtained important coupled fixed point theorems (e.g., [15-20]). In this continuation, Lakshmikantham and Cirić [21] proved coupled common fixed point theorems for nonlinear $\phi$-contraction mappings in partially ordered complete metric spaces which indeed generalize the corresponding fixed point theorems contained in Gnana Bhaskar and Lakshmikantham [22].

As usual, this section is devoted to preliminaries which include basic definitions and results on coupled fixed point for nonlinear contraction mappings defined on partially ordered complete metric spaces. In Section 2, we introduce the concepts of $n$-tupled coincidence point and $n$-tupled fixed point for mappings satisfying different contractive conditions and utilize these two definitions to obtain $n$-tupled coincidence point theorems for nonlinear $\phi$-contraction mappings in partially ordered complete metric spaces.

Now, we present some basic notions and results related to coupled fixed point in metric spaces.

Definition 1 (see [22]). Let $(X, \preceq)$ be a partially ordered set equipped with a metric $d$ such that $(X, d)$ is a metric space. Further, equip the product space $X \times X$ with the following partial ordering:

$$
\begin{gathered}
\text { for }(x, y),(u, v) \in X \times X, \\
\text { define }(u, v) \leq(x, y) \Longleftrightarrow x \geq u, y \leq v .
\end{gathered}
$$

Definition 2 (see [22]). Let $(X, \preceq)$ be a partially ordered set and $F: X \times X \rightarrow X$. One says that $F$ enjoys the mixed monotone property if $F(x, y)$ is monotonically nondecreasing in $x$ and monotonically nonincreasing in $y$; that is, for any $x, y \in X$,

$$
\begin{aligned}
& x_{1}, x_{2} \in X, \quad x_{1} \preceq x_{2} \Longrightarrow F\left(x_{1}, y\right) \preceq F\left(x_{2}, y\right), \\
& y_{1}, y_{2} \in X, \quad y_{1} \preceq y_{2} \Longrightarrow F\left(x, y_{1}\right) \succeq F\left(x, y_{2}\right) .
\end{aligned}
$$

Definition 3 (see [22]). Let $(X, \preceq)$ be a partially ordered set and $F: X \times X \rightarrow X$. One says that $(x, y) \in X \times X$ is a coupled fixed point of the mapping $F$ if

$$
F(x, y)=x, \quad F(y, x)=y .
$$


Theorem 4 (see [22]). Let $(X, \preceq)$ be a partially ordered set equipped with a metric $d$ such that $(X, d)$ is a complete metric space. Let $F: X \times X \rightarrow X$ be a continuous mapping having the mixed monotone property on $X$. Assume that there exists a constant $k \in[0,1)$ with

$$
\begin{aligned}
& d(F(x, y), F(u, v)) \\
& \quad \leq \frac{k}{2}[d(x, u)+d(y, v)] \quad \forall x \geq u, y \leq v .
\end{aligned}
$$

If there exist $x_{0}, y_{0} \in X$ such that $x_{0} \leq F\left(x_{0}, y_{0}\right)$ and $y_{0} \geq$ $F\left(y_{0}, x_{0}\right)$, then there exist $x, y \in X$ such that $x=F(x, y)$ and $y=F(y, x)$.

Definition 5 (see [21]). Let $(X, \preceq)$ be a partially ordered set and $F: X \times X \rightarrow X$ and $g: X \rightarrow X$ two mappings. The mapping $F$ is said to have the mixed $g$-monotone property if $F$ is monotone $g$-nondecreasing in its first argument and is monotone $g$-nonincreasing in its second argument, that is, if, for all $x_{1}, x_{2} \in X, g\left(x_{1}\right) \preceq g\left(x_{2}\right)$ implies $F\left(x_{1}, y\right) \preceq F\left(x_{2}, y\right)$, for any $y \in X$, and, for all $y_{1}, y_{2} \in X, g\left(y_{1}\right) \leq g\left(y_{2}\right)$ implies $F\left(x, y_{1}\right) \geq F\left(x, y_{2}\right)$, for any $x \in X$.

Definition 6 (see [21]). An element $(x, y) \in X \times X$ is called a coupled coincidence point of mappings $F: X \times X \rightarrow X$ and $g: X \rightarrow X$ if

$$
F(x, y)=g(x), \quad F(y, x)=g(y) .
$$

Theorem 7 (see [21]). Let $(X, \preceq)$ be a partially ordered set equipped with a metric $d$ such that $(X, d)$ is a complete metric space. Assume that there is a function $\phi:[0, \infty) \rightarrow[0, \infty)$ with $\phi(t)<t$ and $\lim _{r \rightarrow t^{+}} \phi(r)<t$ for each $t>0$. Let $F: X \times X \rightarrow X$ and $g: X \rightarrow X$ be maps such that $F$ has the mixed $g$-monotone property and

$$
\begin{aligned}
& d(F(x, y), F(u, v)) \\
& \quad \leq \phi\left(\frac{d(g(x), g(u))+d(g(y), g(v))}{2}\right)
\end{aligned}
$$

for all $x, y, u, v \in X$ for which $g(x) \preceq g(u)$ and $g(y) \geq g(v)$. Suppose that $F(X \times X) \subseteq g(X), g$ is continuous and commutes with $F$ besides

(a) $F$ is continuous,

(b) X has the following properties:

(i) if nondecreasing sequence $\left\{x_{n}\right\} \rightarrow x$, then $x_{n} \preceq$ $x$ for all $n$,

(ii) ifnonincreasing sequence $\left\{y_{n}\right\} \rightarrow y$, then $y \leq y_{n}$ for all $n$.

(iii) if a nondecreasing sequence $\left\{x_{n}\right\} \rightarrow x$, then $x_{n} \preceq$ $x$ for all $n \geq 0$,

(iv) if a nonincreasing sequence $\left\{x_{n}\right\} \rightarrow x$, then $x_{n} \geq$ $x$ for all $n \geq 0$.

If there exist $x_{0}, y_{0} \in X$ such that

$$
g\left(x_{0}\right) \preceq F\left(x_{0}, y_{0}\right), \quad g\left(y_{0}\right) \geq F\left(y_{0}, x_{0}\right),
$$

then there exist $x, y \in X$ such that

$$
g(x)=F(x, y), \quad g(y)=F(y, x) .
$$

That is, $F$ and $g$ have a coupled coincidence point.

\section{Main Results}

Throughout the paper, $r$ stands for a general even natural number.

Definition 8. Let $(X, \preceq)$ be a partially ordered set and $F$ : $\prod_{i=1}^{r} X^{i} \rightarrow X$ a mapping. The mapping $F$ is said to have the mixed monotone property if $F$ is nondecreasing in its odd position arguments and nonincreasing in its even position arguments, that is, if,

(i) for all $x_{1}^{1}, x_{2}^{1} \in X, x_{1}^{1} \preceq x_{2}^{1}$ implies $F\left(x_{1}^{1}, x^{2}\right.$, $\left.x^{3}, \ldots, x^{r}\right) \preceq F\left(x_{2}^{1}, x^{2}, x^{3}, \ldots, x^{r}\right)$

(ii) for all $x_{1}^{2}, x_{2}^{2} \in X, x_{1}^{2} \preceq x_{2}^{2}$ implies $F\left(x^{1}, x_{1}^{2}\right.$, $\left.x^{3}, \ldots, x^{r}\right) \geq F\left(x^{1}, x_{2}^{2}, x^{3}, \ldots, x^{r}\right)$

(iii) for all $x_{1}^{3}, x_{2}^{3} \in X, x_{1}^{3} \leq x_{2}^{3}$ implies $F\left(x^{1}, x^{2}\right.$, $\left.x_{1}^{3}, \ldots, x^{r}\right) \preceq F\left(x^{1}, x^{2}, x_{2}^{3}, \ldots, x^{r}\right)$

for all $x_{1}^{r}, x_{2}^{r} \in X, x_{1}^{r} \preceq x_{2}^{r}$ implies $F\left(x^{1}, x^{2}, x^{3}, \ldots, x_{1}^{r}\right) \succeq$ $F\left(x^{1}, x^{2}, x^{3}, \ldots, x_{2}^{r}\right)$.

Definition 9. Let $(X, \preceq)$ be a partially ordered set. Let $F$ : $\prod_{i=1}^{r} X^{i} \rightarrow X$ and $g: X \rightarrow X$ be two mappings. Then the mapping $F$ is said to have the mixed $g$-monotone property if $F$ is $g$-nondecreasing in its odd position arguments and $g$ nonincreasing in its even position arguments, that is, if,

(i) for all $x_{1}^{1}, x_{2}^{1} \in X, g x_{1}^{1} \preceq g x_{2}^{1}$ implies $F\left(x_{1}^{1}, x^{2}\right.$, $\left.x^{3}, \ldots, x^{r}\right) \preceq F\left(x_{2}^{1}, x^{2}, x^{3}, \ldots, x^{r}\right)$,

(ii) for all $x_{1}^{2}, x_{2}^{2} \in X, g x_{1}^{2} \preceq g x_{2}^{2}$ implies $F\left(x^{1}, x_{1}^{2}\right.$, $\left.x^{3}, \ldots, x^{r}\right) \geq F\left(x^{1}, x_{2}^{2}, x^{3}, \ldots, x^{r}\right)$,

(iii) for all $x_{1}^{3}, x_{2}^{3} \in X, g x_{1}^{3} \preceq g x_{2}^{3}$ implies $F\left(x^{1}, x^{2}\right.$, $\left.x_{1}^{3}, \ldots, x^{r}\right) \leq F\left(x^{1}, x^{2}, x_{2}^{3}, \ldots, x^{r}\right)$,

for all $x_{1}^{r}, x_{2}^{r} \in X, g x_{1}^{r} \preceq g x_{2}^{r}$ implies $F\left(x^{1}, x^{2}, x^{3}, \ldots, x_{1}^{r}\right) \geq$ $F\left(x^{1}, x^{2}, x^{3}, \ldots, x_{2}^{r}\right)$.

Definition 10. Let $X$ be a nonempty set. An element $\left(x^{1}, x^{2}, x^{3}, \ldots, x^{r}\right) \in \prod_{i=1}^{r} X^{i}$ is called an $r$-tupled fixed point of the mapping $F: \prod_{i=1}^{r} X^{i} \rightarrow X$ if

$$
\begin{aligned}
x^{1} & =F\left(x^{1}, x^{2}, x^{3}, \ldots, x^{r}\right), \\
x^{2} & =F\left(x^{2}, x^{3}, \ldots, x^{r}, x^{1}\right), \\
x^{3} & =F\left(x^{3}, \ldots, x^{r}, x^{1}, x^{2}\right), \\
& \vdots \\
x^{r} & =F\left(x^{r}, x^{1}, x^{2}, \ldots, x^{r-1}\right) .
\end{aligned}
$$


Definition 11. Let $X$ be a nonempty set. An element $\left(x^{1}, x^{2}, x^{3}, \ldots, x^{r}\right) \in \prod_{i=1}^{r} X^{i}$ is called an $r$-tupled coincidence point of the mappings $F: \prod_{i=1}^{r} X^{i} \rightarrow X$ and $g: X \rightarrow X$ if

$$
\begin{aligned}
g x^{1} & =F\left(x^{1}, x^{2}, x^{3}, \ldots, x^{r}\right), \\
g x^{2} & =F\left(x^{2}, x^{3}, \ldots, x^{r}, x^{1}\right), \\
g x^{3} & =F\left(x^{3}, \ldots, x^{r}, x^{1}, x^{2}\right), \\
& \vdots \\
g x^{r} & =F\left(x^{r}, x^{1}, x^{2}, \ldots, x^{r-1}\right) .
\end{aligned}
$$

Definition 12. Let $X$ be a nonempty set. The mappings $F$ : $\prod_{i=1}^{r} X^{i} \rightarrow X$ and $g: X \rightarrow X$ are said to be commutating if

$$
g\left(F\left(x^{1}, x^{2}, \ldots, x^{r}\right)\right)=\left(F\left(g\left(x^{1}\right), g\left(x^{2}\right), \ldots, g\left(x^{r}\right)\right)\right.
$$

for all $x^{1}, x^{2}, \ldots, x^{r} \in X$.

Now, we are equipped to prove our main result as follows.

Theorem 13. Let $(X, \preceq)$ be a partially ordered set equipped with a metric $d$ such that $(X, d)$ is a complete metric space. Assume that there is a function $\phi:[0,+\infty) \rightarrow[0,+\infty)$ with $\phi(t)<t$ and $\lim _{r \rightarrow t^{+}} \phi(r)<t$ for each $t>0$. Further, suppose that $F: \prod_{i=1}^{r} X^{i} \rightarrow X$ and $g: X \rightarrow X$ are two maps such that $F$ has the mixed $g$-monotone property satisfying the following conditions:

(i) $F\left(\prod_{i=1}^{r} X^{i}\right) \subseteq g(X)$,

(ii) $g$ is continuous and monotonically increasing,

(iii) $(g, F)$ is a commutating pair,

(iv)

$$
\begin{gathered}
d\left(F\left(x^{1}, x^{2}, \ldots, x^{r}\right), F\left(y^{1}, y^{2}, \ldots, y^{r}\right)\right) \\
\leq \phi\left(\frac{1}{r} \sum_{n=1}^{r} d\left(g\left(x^{n}\right), g\left(y^{n}\right)\right)\right),
\end{gathered}
$$

for all $x^{1}, x^{2}, x^{3}, \ldots, x^{r}, y^{1}, y^{2}, y^{3}, \ldots, y^{r} \in X$, with $g x^{1} \leq$ $g y^{1}, g x^{2} \geq g y^{2}, g x^{3} \leq g y^{3}, \ldots, g x^{r} \geq g y^{r}$. Also, suppose that either

(a) $F$ is continuous or

(b) X has the following properties:

(i) if a nondecreasing sequence $\left\{x_{n}\right\} \longrightarrow x$,

$$
\text { then } x_{n} \preceq x \quad \forall n \geq 0,
$$

(ii) if a nonincreasing sequence $\left\{x_{n}\right\} \longrightarrow x$,

$$
\text { then } x_{n} \geq x \quad \forall n \geq 0 .
$$

If there exist $x_{0}^{1}, x_{0}^{2}, x_{0}^{3}, \ldots, x_{0}^{r} \in X$ such that

$$
\begin{aligned}
g x_{0}^{1} & \leq F\left(x_{0}^{1}, x_{0}^{2}, x_{0}^{3}, \ldots, x_{0}^{r}\right), \\
g x_{0}^{2} & \geq F\left(x_{0}^{2}, x_{0}^{3}, \ldots, x_{0}^{r}, x_{0}^{1}\right), \\
g x_{0}^{3} & \leq F\left(x_{0}^{3}, \ldots, x_{0}^{r}, x_{0}^{1}, x_{0}^{2}\right), \\
& \vdots \\
g x_{0}^{r} & \geq F\left(x_{0}^{r}, x_{0}^{1}, x_{0}^{2}, \ldots, x_{0}^{r-1}\right),
\end{aligned}
$$

then $F$ and $g$ have a $r$-tupled coincidence point; that is, there exist $x^{1}, x^{2}, x^{3}, \ldots, x^{r} \in X$ such that

$$
\begin{aligned}
g x^{1} & =F\left(x^{1}, x^{2}, x^{3}, \ldots, x^{r}\right), \\
g x^{2} & =F\left(x^{2}, x^{3}, \ldots, x^{r}, x^{1}\right), \\
g x^{3} & =F\left(x^{3}, \ldots, x^{r}, x^{1}, x^{2}\right), \\
& \vdots \\
g x^{r} & =F\left(x^{r}, x^{1}, x^{2}, x^{3}, \ldots, x^{r-1}\right) .
\end{aligned}
$$

Proof. Starting with $x_{0}^{1}, x_{0}^{2}, x_{0}^{3}, \ldots, x_{0}^{r}$ in $X$, we define the sequences $\left\{x_{n}^{1}\right\},\left\{x_{n}^{2}\right\},\left\{x_{n}^{3}\right\}, \ldots,\left\{x_{n}^{r}\right\}$ in $X$ as follows:

$$
\begin{aligned}
& g x_{n+1}^{1}=F\left(x_{n}^{1}, x_{n}^{2}, x_{n}^{3}, \ldots, x_{n}^{r}\right), \\
& g x_{n+1}^{2}=F\left(x_{n}^{2}, x_{n}^{3}, \ldots, x_{n}^{r}, x_{n}^{1}\right), \\
& g x_{n+1}^{3}=F\left(x_{n}^{3}, \ldots, x_{n}^{r}, x_{n}^{1}, x_{n}^{2}\right),
\end{aligned}
$$

$$
g x_{n+1}^{r}=F\left(x_{n}^{r}, x_{n}^{1}, x_{n}^{2}, x_{n}^{3}, \ldots, x_{n}^{r-1}\right) \text {. }
$$

Now, we prove that for all $n \geq 0$,

$$
\begin{gathered}
g x_{n}^{1} \preceq g x_{n+1}^{1}, \quad g x_{n}^{2} \geq g x_{n+1}^{2}, \\
g x_{n}^{3} \preceq g x_{n+1}^{3}, \ldots, g x_{n}^{r} \geq g x_{n+1}^{r} . \\
g x_{0}^{1} \leq F\left(x_{0}^{1}, x_{0}^{2}, x_{0}^{3}, \ldots, x_{0}^{r}\right)=x_{1}^{1}, \\
g x_{0}^{2} \succeq F\left(x_{0}^{2}, x_{0}^{3}, \ldots, x_{0}^{r}, x_{0}^{1}\right)=x_{1}^{2}, \\
g x_{0}^{3} \leq F\left(x_{0}^{3}, \ldots, x_{0}^{r}, x_{0}^{1}, x_{0}^{2}\right)=x_{1}^{3},
\end{gathered}
$$

$g x_{0}^{r} \geq F\left(x_{0}^{r}, x_{0}^{1}, x_{0}^{2}, x_{0}^{3}, \ldots, x_{0}^{r-1}\right)=x_{1}^{r}$. 
So (17) holds for $n=0$. Suppose (17) holds for some $n>0$. Consider

$$
\begin{aligned}
& g x_{n+1}^{1}=F\left(x_{n}^{1}, x_{n}^{2}, x_{n}^{3}, \ldots, x_{n}^{r}\right) \\
& \preceq F\left(x_{n+1}^{1}, x_{n}^{2}, x_{n}^{3}, \ldots, x_{n}^{r}\right) \\
& \preceq F\left(x_{n+1}^{1}, x_{n+1}^{2}, x_{n}^{3}, \ldots, x_{n}^{r}\right) \\
& \preceq F\left(x_{n+1}^{1}, x_{n+1}^{2}, x_{n+1}^{3}, \ldots, x_{n}^{r}\right) \\
& \preceq F\left(x_{n+1}^{1}, x_{n+1}^{2}, x_{n+1}^{3}, \ldots, x_{n+1}^{r}\right) \\
& =g x_{n+2}^{1} \text {, } \\
& g x_{n+1}^{2}=F\left(x_{n}^{2}, x_{n}^{3}, \ldots, x_{n}^{r}, x_{n}^{1}\right) \\
& \succeq F\left(x_{n+1}^{2}, x_{n}^{3}, \ldots, x_{n}^{r}, x_{n}^{1}\right) \\
& \geq F\left(x_{n+1}^{2}, x_{n+1}^{3}, \ldots, x_{n}^{r}, x_{n}^{1}\right) \\
& \succeq F\left(x_{n+1}^{2}, x_{n+1}^{3}, \ldots, x_{n+1}^{r}, x_{n}^{1}\right) \\
& \succeq F\left(x_{n+1}^{2}, x_{n+1}^{3}, \ldots, x_{n+1}^{r}, x_{n+1}^{1}\right) \\
& =g x_{n+2}^{2} \text {, } \\
& g x_{n+1}^{3}=F\left(x_{n}^{3}, \ldots, x_{n}^{r}, x_{n}^{1}, x_{n}^{2}\right) \\
& \preceq F\left(x_{n+1}^{3}, \ldots, x_{n}^{r}, x_{n}^{1}, x_{n}^{2}\right) \\
& \preceq F\left(x_{n+1}^{3}, \ldots, x_{n+1}^{r}, x_{n}^{1}, x_{n}^{2}\right) \\
& \preceq F\left(x_{n+1}^{3}, \ldots, x_{n+1}^{r}, x_{n+1}^{1}, x_{n}^{2}\right) \\
& \preceq F\left(x_{n+1}^{3}, \ldots, x_{n+1}^{r}, x_{n+1}^{1}, x_{n+1}^{2}\right) \\
& =g x_{n+2}^{3} \text {, } \\
& g x_{n+1}^{r}=F\left(x_{n}^{r}, x_{n}^{1}, x_{n}^{2}, x_{n}^{3}, \ldots, x_{n}^{r-1}\right) \\
& \geq F\left(x_{n+1}^{r}, x_{n}^{1}, x_{n}^{2}, x_{n}^{3}, \ldots, x_{n}^{r-1}\right) \\
& \succeq F\left(x_{n+1}^{r}, x_{n+1}^{1}, x_{n}^{2}, x_{n}^{3}, \ldots, x_{n}^{r-1}\right) \\
& \succeq F\left(x_{n+1}^{r}, x_{n+1}^{1}, x_{n+1}^{2}, x_{n}^{3}, \ldots, x_{n}^{r-1}\right) \\
& \succeq F\left(x_{n+1}^{r}, x_{n+1}^{1}, x_{n+1}^{2}, x_{n+1}^{3}, \ldots, x_{n}^{r-1}\right) \\
& \succeq F\left(x_{n+1}^{r}, x_{n+1}^{1}, x_{n+1}^{2}, x_{n+1}^{3}, \ldots, x_{n+1}^{r-1}\right) \\
& =g x_{n+2}^{r} \text {. }
\end{aligned}
$$

$$
\begin{aligned}
\delta_{m}= & d\left(g\left(x_{m}^{1}\right), g\left(x_{m+1}^{1}\right)\right)+d\left(g\left(x_{m}^{2}\right), g\left(x_{m+1}^{2}\right)\right) \\
& +\cdots+d\left(g\left(x_{m}^{r}\right), g\left(x_{m+1}^{r}\right)\right) \\
\leq & r \phi\left(\frac{1}{r} \sum_{n=1}^{r} d\left(g\left(x_{m-1}^{n}\right), g\left(x_{m}^{n}\right)\right)\right) \\
= & r \phi\left(\frac{1}{r} \delta_{m-1}\right) .
\end{aligned}
$$

Since $\phi(t)<t$ for all $t>0$, therefore, $\delta_{m} \leq \delta_{m-1}$ for all $m$ so that $\left\{\delta_{m}\right\}$ is a nonincreasing sequence. Since it is bounded below, there is some $\delta \geq 0$ such that

$$
\lim _{m \rightarrow \infty} \delta_{m}=+\delta \text {. }
$$

We shall show that $\delta=0$. Suppose, on the contrary that $\delta>0$. Taking the limits as $m \rightarrow+\infty$ of both the sides of (23) and keeping in mind our supposition that $\lim _{r \rightarrow t^{+}} \phi(r)<t$ for all $t>0$, we have

$$
\delta=\lim _{m \rightarrow \infty} \delta_{m} \leq \lim _{m \rightarrow \infty} r \phi\left(\frac{1}{r} \delta_{m-1}\right)=r \phi\left(\frac{1}{r} \delta\right)<r \frac{\delta}{r}=\delta,
$$

which is a contradiction so that $\delta=0$ yielding thereby

$$
\begin{gathered}
\lim _{m \rightarrow \infty} d\left(g\left(x_{m}^{1}\right), g\left(x_{m+1}^{1}\right)\right)+d\left(g\left(x_{m}^{2}\right), g\left(x_{m+1}^{2}\right)\right) \\
+\cdots+d\left(g\left(x_{m}^{r}\right), g\left(x_{m+1}^{r}\right)\right)=0 .
\end{gathered}
$$


Next we show that all the sequences $\left\{g\left(x_{m}^{1}\right)\right\},\left\{g\left(x_{m}^{2}\right)\right\}, \ldots$, and $\left\{g\left(x_{m}^{r}\right)\right\}$ are Cauchy sequences. If possible, suppose that at least one of $\left\{g\left(x_{m}^{1}\right)\right\},\left\{g\left(x_{m}^{2}\right)\right\}, \ldots$ and $\left\{g\left(x_{m}^{r}\right)\right\}$ is not a Cauchy sequence. Then there exists $\epsilon>0$ and sequences of positive integers $\{l(k)\}$ and $\{m(k)\}$ such that for all positive integers $k$,

$$
\begin{gathered}
m(k)>l(k)>k, \\
d\left(g x_{l(k)}^{1}, g x_{m(k)}^{1}\right)+d\left(g x_{l(k)}^{2}, g x_{m(k)}^{2}\right) \\
+\cdots+d\left(g x_{l(k)}^{r}, g x_{m(k)}^{r}\right) \geq \epsilon, \\
d\left(g x_{l(k)}^{1}, g x_{m(k)-1}^{1}\right)+d\left(g x_{l(k)}^{2}, g x_{m(k)-1}^{2}\right) \\
+\cdots+d\left(g x_{l(k)}^{r}, g x_{m(k)-1}^{r}\right)<\epsilon .
\end{gathered}
$$

Now,

$$
\begin{aligned}
\epsilon \leq & d\left(g x_{l(k)}^{1}, g x_{m(k)}^{1}\right)+d\left(g x_{l(k)}^{2}, g x_{m(k)}^{2}\right) \\
& +\cdots+d\left(g x_{l(k)}^{r}, g x_{m(k)}^{r}\right) \\
\leq & d\left(g x_{l(k)}^{1}, g x_{m(k)-1}^{1}\right)+d\left(g x_{l(k)}^{2}, g x_{m(k)-1}^{2}\right) \\
& +\cdots+d\left(g x_{l(k)}^{r}, g x_{m(k)-1}^{r}\right)+d\left(g x_{m(k)-1}^{1}, g x_{m(k)}^{1}\right) \\
& +d\left(g x_{m(k)-1}^{2}, g x_{m(k)}^{2}\right)+\cdots+d\left(g x_{m(k)-1}^{r}, g x_{m(k)}^{r}\right),
\end{aligned}
$$

that is,

$$
\begin{aligned}
\epsilon \leq & d\left(g x_{l(k)}^{1}, g x_{m(k)}^{1}\right)+d\left(g x_{l(k)}^{2}, g x_{m(k)}^{2}\right) \\
& +\cdots+d\left(g x_{l(k)}^{r}, g x_{m(k)}^{r}\right) \\
\leq & \epsilon+d\left(g x_{m(k)-1}^{1}, g x_{m(k)}^{1}\right) \\
& +d\left(g x_{m(k)-1}^{2}, g x_{m(k)}^{2}\right)+\cdots+d\left(g x_{m(k)-1}^{r}, g x_{m(k)}^{r}\right) .
\end{aligned}
$$

Letting $k \rightarrow \infty$ in the above inequality and using (26), we have

$$
\begin{gathered}
\lim _{k \rightarrow \infty} d\left(g x_{l(k)}^{1}, g x_{m(k)}^{1}\right)+d\left(g x_{l(k)}^{2}, g x_{m(k)}^{2}\right) \\
+\cdots+d\left(g x_{l(k)}^{r}, g x_{m(k)}^{r}\right)=\epsilon .
\end{gathered}
$$

Again,

$$
\begin{gathered}
d\left(g x_{l(k)+1}^{1}, g x_{m(k)+1}^{1}\right)+d\left(g x_{l(k)+1}^{2}, g x_{m(k)+1}^{2}\right) \\
+\cdots+d\left(g x_{l(k)+1}^{r}, g x_{m(k)+1}^{r}\right)
\end{gathered}
$$

$$
\begin{aligned}
\leq & d\left(g x_{l(k)+1}^{1}, g x_{l(k)}^{1}\right)+d\left(g x_{l(k)+1}^{2}, g x_{l(k)}^{2}\right) \\
& +\cdots+d\left(g x_{l(k)+1}^{r}, g x_{l(k)}^{r}\right)+d\left(g x_{l(k)}^{1}, g x_{m(k)}^{1}\right) \\
& +d\left(g x_{l(k)}^{2}, g x_{m(k)}^{2}\right)+\cdots+d\left(g x_{l(k)}^{r}, g x_{m(k)}^{r}\right) \\
& +d\left(g x_{m(k)}^{1}, g x_{m(k)+1}^{1}\right)+d\left(g x_{m(k)}^{2}, g x_{m(k)+1}^{2}\right) \\
& +\cdots+d\left(g x_{m(k)}^{r}, g x_{m(k)+1}^{r}\right),
\end{aligned}
$$

$$
\begin{aligned}
d\left(g x_{l(k)}^{1}, g x_{m(k)}^{1}\right)+d\left(g x_{l(k)}^{2}, g x_{m(k)}^{2}\right) \\
\quad+\cdots+d\left(g x_{l(k)}^{r}, g x_{m(k)}^{r}\right) \leq d\left(g x_{l(k)+1}^{1}, g x_{l(k)}^{1}\right) \\
\quad+d\left(g x_{l(k)+1}^{2}, g x_{l(k)}^{2}\right)+\cdots+d\left(g x_{l(k)+1}^{r}, g x_{l(k)}^{r}\right) \\
\quad+d\left(g x_{l(k)+1}^{1}, g x_{m(k)+1}^{1}\right)+d\left(g x_{l(k)+1}^{2}, g x_{m(k)+1}^{2}\right) \\
\quad+\cdots+d\left(g x_{l(k)+1}^{r}, g x_{m(k)+1}^{r}\right)+d\left(g x_{m(k)}^{1}, g x_{m(k)+1}^{1}\right) \\
\quad+d\left(g x_{m(k)}^{2}, g x_{m(k)+1}^{2}\right)+\cdots+d\left(g x_{m(k)}^{r}, g x_{m(k)+1}^{r}\right) .
\end{aligned}
$$

Letting $k \rightarrow \infty$ in the above inequalities, using (26) and (30), we have

$$
\begin{gathered}
\lim _{k \rightarrow \infty}\left\{d\left(g x_{l(k)+1}^{1}, g x_{m(k)+1}^{1}\right)+d\left(g x_{l(k)+1}^{2}, g x_{m(k)+1}^{2}\right)\right. \\
\left.+\cdots+d\left(g x_{l(k)+1}^{r}, g x_{m(k)+1}^{r}\right)\right\}=\epsilon .
\end{gathered}
$$

Now,

$$
\begin{gathered}
d\left(g x_{l(k)+1}^{1}, g x_{m(k)+1}^{1}\right)+d\left(g x_{l(k)+1}^{2}, g x_{m(k)+1}^{2}\right) \\
+\cdots+d\left(g x_{l(k)+1}^{r}, g x_{m(k)+1}^{r}\right) \\
=d\left(F\left(x_{l(k)}^{1}, x_{l(k)}^{2}, \ldots, x_{l(k)}^{r}\right),\right. \\
\left.F\left(x_{m(k)}^{1}, x_{m(k)}^{2}, \ldots, x_{m(k)}^{r}\right)\right) \\
+d\left(F\left(x_{l(k)}^{2}, x_{l(k)}^{3}, \ldots, x_{l(k)}^{r}, x_{l(k)}^{1}\right),\right. \\
\left.F\left(x_{m(k)}^{2}, x_{m(k)}^{3}, \ldots, x_{m(k)}^{r}, x_{l(k)}^{1}\right)\right) \ldots \\
+d\left(F\left(x_{l(k)}^{r}, x_{l(k)}^{1}, \ldots, x_{l(k)}^{r-1}\right),\right. \\
\left.F\left(x_{m(k)}^{r}, x_{m(k)}^{1}, \ldots, x_{m(k)}^{r-1}\right)\right) \\
\leq r \phi\left(\frac{1}{r} \sum_{n=1}^{r} d\left(g\left(x_{l(k)}^{n}\right), g\left(x_{m(k)}^{n}\right)\right)\right) .
\end{gathered}
$$

Letting $k \rightarrow \infty$ in the above inequality, using (30), (33), and the property of $\phi$, we have

$$
\epsilon \leq r \phi\left(\frac{\epsilon}{r}\right)<r \frac{\epsilon}{r}=\epsilon,
$$

which is a contradiction. Therefore, $\left\{g\left(x_{m}^{1}\right)\right\},\left\{g\left(x_{m}^{2}\right)\right\}, \ldots$, and $\left\{g\left(x_{m}^{r}\right)\right\}$ are Cauchy sequences in $(X, d)$. Since the metric 
space $(X, d)$ is complete, so there exist $x^{1}, x^{2}, \ldots, x^{r} \in X$ such that

$$
\begin{gathered}
\lim _{m \rightarrow \infty} g\left(x_{m}^{1}\right)=x^{1}, \\
\lim _{m \rightarrow \infty} g\left(x_{m}^{2}\right)=x^{2}, \ldots, \\
\lim _{m \rightarrow \infty} g\left(x_{m}^{r}\right)=x^{r} .
\end{gathered}
$$

By the continuity of $g$ and (36), we can have

$$
\begin{gathered}
\lim _{m \rightarrow \infty} g\left(g\left(x_{m}^{1}\right)\right)=g\left(x^{1}\right), \\
\lim _{m \rightarrow \infty} g\left(g\left(x_{m}^{2}\right)\right)=g\left(x^{2}\right), \ldots, \\
\lim _{m \rightarrow \infty} g\left(g\left(x_{m}^{r}\right)\right)=g\left(x^{r}\right) .
\end{gathered}
$$

Using (16) and the commutativity of $F$ with $g$, we get

$$
\begin{aligned}
g\left(g\left(x_{m+1}^{1}\right)\right) & =g\left(F\left(x_{m}^{1}, x_{m}^{2}, \ldots, x_{m}^{r}\right)\right) \\
& =F\left(g\left(x_{m}^{1}\right), g\left(x_{m}^{2}\right), \ldots, g\left(x_{m}^{r}\right)\right), \\
g\left(g\left(x_{m+1}^{2}\right)\right) & =g\left(F\left(x_{m}^{2}, x_{m}^{3}, \ldots, x_{m}^{r}\right)\right) \\
& =F\left(g\left(x_{m}^{2}\right), g\left(x_{m}^{3}\right), \ldots, g\left(x_{m}^{1}\right)\right), \\
& \vdots \\
g\left(g\left(x_{m+1}^{r}\right)\right) & =g\left(F\left(x_{m}^{r}, x_{m}^{1}, \ldots, x_{m}^{r-1}\right)\right) \\
& =F\left(g\left(x_{m}^{r}\right), g\left(x_{m}^{1}\right), \ldots, g\left(x_{m}^{r-1}\right)\right) .
\end{aligned}
$$

Now, we show that $F$ and $g$ have an $r$-tupled coincidence point. To accomplish this, suppose (a) holds, then using (16), (37), and the continuities of $F$ and $g$, we obtain

$$
\begin{aligned}
g\left(x^{1}\right) & =\lim _{m \rightarrow \infty} g\left(g\left(x_{m+1}^{1}\right)\right)=\lim _{m \rightarrow \infty} g\left(F\left(x_{m}^{1}, x_{m}^{2}, \ldots, x_{m}^{r}\right)\right) \\
& =F\left(\lim _{m \rightarrow \infty} g\left(x_{m}^{1}\right), \lim _{m \rightarrow \infty} g\left(x_{m}^{2}\right), \ldots, \lim _{m \rightarrow \infty} g\left(x_{m}^{r}\right)\right) \\
& =F\left(x^{1}, x^{2}, \ldots, x^{r}\right) .
\end{aligned}
$$

Similarly, we can also show that

$$
\begin{aligned}
g\left(x^{2}\right) & =F\left(x^{2}, x^{3}, \ldots, x^{r}, x^{1}\right), \\
g\left(x^{3}\right) & =F\left(x^{3}, \ldots, x^{r}, x^{1}, x^{2}\right), \\
& \vdots \\
g\left(x^{r}\right) & =F\left(x^{r}, x^{1}, \ldots, x^{r-1}\right) .
\end{aligned}
$$

Hence the element $\left(x^{1}, x^{2}, \ldots, x^{r}\right) \in \prod_{i=1}^{r} X^{i}$ is a $r$-tupled coincidence point of the mappings $F$ and $g$. Next, assume that (b) holds. Since $\left\{g\left(x_{m}^{i}\right)\right\}$ is nondecreasing or nonincreasing as $i$ is odd or even and $g\left(x_{m}^{i}\right) \rightarrow x^{i}$ as $m \rightarrow \infty$, we have $g\left(x_{m}^{i}\right) \leq x^{i}$, when $i$ is odd while $g\left(x_{m}^{i}\right) \geq x^{i}$, when $i$ is even.

Since $g$ is monotonically increasing, therefore

$$
\begin{aligned}
& g\left(g\left(x_{m}^{i}\right)\right) \leq g\left(x^{i}\right) \text { when } i \text { is odd, } \\
& g\left(g\left(x_{m}^{i}\right)\right) \geq g\left(x^{i}\right) \text { when } i \text { is even. }
\end{aligned}
$$

On using triangle inequality together with (16), we get

$$
\begin{aligned}
& d\left(g\left(x^{1}\right), F\left(x^{1}, \ldots, x^{r}\right)\right) \\
& \leq d\left(g\left(x^{1}\right), g\left(g\left(x_{m+1}^{1}\right)\right)\right) \\
&+d\left(g\left(g\left(x_{m+1}^{1}\right)\right), F\left(x^{1}, \ldots, x^{r}\right)\right) \\
& \leq d\left(g\left(x^{1}\right), g\left(g\left(x_{m+1}^{1}\right)\right)\right) \\
& \quad+\phi\left(\frac{1}{r} \sum_{n=1}^{r} d\left(g\left(g\left(x_{m}^{n}\right)\right), g\left(x^{n}\right)\right)\right) .
\end{aligned}
$$

Letting $m \rightarrow \infty$ in the above inequality and using (37), we have $g\left(x^{1}\right)=F\left(x^{1}, x^{2} \ldots, x^{r}\right)$. Similarly, we can also show that

$$
g\left(x^{2}\right)=F\left(x^{2}, x^{3} \ldots, x^{1}\right), \ldots, g\left(x^{r}\right)=F\left(x^{r}, x^{1} \ldots, x^{r-1}\right)
$$

which shows that $F$ and $g$ have an $r$-tupled coincidence point. This completes the proof.

Corollary 14. Let $(X, \preceq)$ be a partially ordered set equipped with a metric $d$ such that $(X, d)$ is a complete metric space. Assume that there is a function $\phi:[0,+\infty) \rightarrow[0,+\infty)$ with $\phi(t)<t$ and $\lim _{r \rightarrow t^{+}} \phi(r)<t$ for each $t>0$. Further, suppose that $F: \prod_{i=1}^{r} X^{i} \rightarrow X$ is a mapping such that $F$ has the mixed monotone property satisfying the following conditions:

$$
\begin{aligned}
& d\left(F\left(x^{1}, x^{2}, \ldots, x^{r}\right), F\left(y^{1}, y^{2}, \ldots, y^{r}\right)\right) \\
& \quad \leq \phi\left(\frac{1}{r} \sum_{n=1}^{r} d\left(x^{n}, y^{n}\right)\right),
\end{aligned}
$$

for all $x^{1}, x^{2}, x^{3}, \ldots, x^{r}, y^{1}, y^{2}, y^{3}, \ldots, y^{r} \in X$ with $x^{1} \preceq$ $y^{1}, x^{2} \geq y^{2}, x^{3} \leq y^{3}, \ldots, x^{r} \geq y^{r}$. Also, suppose that either
(a) $F$ is continuous or
(b) X has the following properties:

(i) if a nondecreasing sequence $\left\{x_{n}\right\} \rightarrow x$, then $x_{n} \preceq$ $x$ for all $n \geq 0$,

(ii) if a nonincreasing sequence $\left\{x_{n}\right\} \rightarrow x$, then $x_{n} \geq$ $x$ for all $n \geq 0$. 
If there exist $x_{0}^{1}, x_{0}^{2}, x_{0}^{3}, \ldots, x_{0}^{r} \in X$ such that

$$
\begin{aligned}
x_{0}^{1} & \preceq F\left(x_{0}^{1}, x_{0}^{2}, x_{0}^{3}, \ldots, x_{0}^{r}\right), \\
x_{0}^{2} & \succeq F\left(x_{0}^{2}, x_{0}^{3}, \ldots, x_{0}^{r}, x_{0}^{1}\right), \\
x_{0}^{3} & \preceq F\left(x_{0}^{3}, \ldots, x_{0}^{r}, x_{0}^{1}, x_{0}^{2}\right), \\
\vdots & \\
x_{0}^{r} & \geq F\left(x_{0}^{r}, x_{0}^{1}, x_{0}^{2}, \ldots, x_{0}^{r-1}\right),
\end{aligned}
$$

then $F$ has an $r$-tupled fixed point in $X$; that is, there exist $x^{1}, x^{2}, x^{3}, \ldots, x^{r} \in X$ such that

$$
\begin{aligned}
x^{1} & =F\left(x^{1}, x^{2}, x^{3}, \ldots, x^{r}\right), \\
x^{2} & =F\left(x^{2}, x^{3}, \ldots, x^{r}, x^{1}\right), \\
x^{3} & =F\left(x^{3}, \ldots, x^{r}, x^{1}, x^{2}\right), \\
& \vdots \\
x^{r} & =F\left(x^{r}, x^{1}, x^{2}, x^{3}, \ldots, x^{r-1}\right) .
\end{aligned}
$$

Proof. Setting $g=I$, the identity mapping, in Theorem 13, we obtain Corollary 14.

Also, Theorem 13 immediately yields the following corollary.

Corollary 15. Let $(X, \preceq)$ be a partially ordered set equipped with a metric $d$ such that $(X, d)$ is a complete metric space. Suppose that $F: \prod_{i=1}^{r} X^{i} \rightarrow X$ and $g: X \rightarrow X$ are two maps such that $F$ has the mixed $g$-monotone property satisfying the following conditions:

(i) $F\left(\prod_{i=1}^{r} X^{i}\right) \subseteq g(X)$,

(ii) $g$ is continuous and monotonically increasing,

(iii) $(g, F)$ is a commutating pair,

(iv) $d\left(F\left(x^{1}, x^{2}, \ldots, x^{r}\right), F\left(y^{1}, y^{2}, \ldots, y^{r}\right)\right) \leq$ $\times \sum_{n=1}^{r} d\left(g\left(x^{n}\right), g\left(y^{n}\right)\right), k \in[0,1)$

for all $x^{1}, x^{2}, x^{3}, \ldots, x^{r}, y^{1}, y^{2}, y^{3}, \ldots, y^{r} \in X$ with $g x^{1} \preceq$ $g y^{1}, g x^{2} \succeq g y^{2}, g x^{3} \preceq g y^{3}, \ldots, g x^{r} \geq g y^{r}$. Also, suppose that either

(a) $F$ is continuous or

(b) X has the following properties:

(i) if a nondecreasing sequence $\left\{x_{n}\right\} \rightarrow x$, then $x_{n} \preceq$ $x$ for all $n \geq 0$,

(ii) if a nonincreasing sequence $\left\{x_{n}\right\} \rightarrow x$, then $x_{n} \geq$ $x$ for all $n \geq 0$.
If there exist $x_{0}^{1}, x_{0}^{2}, x_{0}^{3}, \ldots, x_{0}^{r} \in X$ such that

$$
\begin{aligned}
g x_{0}^{1} & \leq F\left(x_{0}^{1}, x_{0}^{2}, x_{0}^{3}, \ldots, x_{0}^{r}\right), \\
g x_{0}^{2} & \succeq F\left(x_{0}^{2}, x_{0}^{3}, \ldots, x_{0}^{r}, x_{0}^{1}\right), \\
g x_{0}^{3} & \preceq F\left(x_{0}^{3}, \ldots, x_{0}^{r}, x_{0}^{1}, x_{0}^{2}\right), \\
\vdots & \\
g x_{0}^{r} & \succeq F\left(x_{0}^{r}, x_{0}^{1}, x_{0}^{2}, \ldots, x_{0}^{r-1}\right),
\end{aligned}
$$

then $F$ and $g$ have an $r$-tupled coincidence point in $X$; that is, there exist $x^{1}, x^{2}, x^{3}, \ldots, x^{r} \in X$ such that

$$
\begin{aligned}
g x^{1} & =F\left(x^{1}, x^{2}, x^{3}, \ldots, x^{r}\right), \\
g x^{2} & =F\left(x^{2}, x^{3}, \ldots, x^{r}, x^{1}\right), \\
g x^{3} & =F\left(x^{3}, \ldots, x^{r}, x^{1}, x^{2}\right), \\
& \vdots \\
g x^{r} & =F\left(x^{r}, x^{1}, x^{2}, x^{3}, \ldots, x^{r-1}\right) .
\end{aligned}
$$

Proof. Setting $\phi(t)=k \cdot t$ with $k \in[0,1)$ in Theorem 13, we obtain Corollary 15.

The following example illustrates Theorem 13.

Example 16. Let $X=[0,1]$. Then $X$ is a complete metric space under natural ordering $\preceq$ of real numbers and natural metric $d(x, y)=|x-y|$ for all $x, y \in X$. Define $g: X \rightarrow X$ as $g(x)=x /(r-1)$ wherein $r$ is fixed and $r>1$ for all $x \in X$. Also, define $F: \prod_{i=1}^{r} X^{i} \rightarrow X$ by

$$
F\left(x^{1}, x^{2}, \ldots, x^{r}\right)=\frac{x^{1}-x^{2}+x^{3}-\cdots+x^{r-1}-x^{r}}{r^{2}-1}
$$

for all $x^{1}, x^{2}, \ldots, x^{r} \in X$. Define $\phi:[0, \infty) \rightarrow[0, \infty)$ as $\phi(t)=(r /(r+1)) t$, where $r$ is fixed as earlier. Then $\phi$ has all the properties mentioned in Theorem 13. Also $F$ and $g$ are commutating mapping in $X$.

Next, we verify inequality (12) (of Theorem 13)

$$
\begin{array}{r}
d\left(F\left(x^{1}, x^{2}, \ldots, x^{r}\right), F\left(y^{1}, y^{2}, \ldots, y^{r}\right)\right) \\
=d\left(\frac{x^{1}-x^{2}+x^{3}-x^{4}+\cdots+x^{r-1}-x^{r}}{r^{2}-1},\right. \\
\left.\frac{y^{1}-y^{2}+y^{3}-y^{4}+\cdots+y^{r-1}-y^{r}}{r^{2}-1}\right)
\end{array}
$$




$$
\begin{aligned}
& =\frac{1}{r+1} \mid \frac{x^{1}-x^{2}+x^{3}-x^{4}+\cdots+x^{r-1}-x^{r}}{r-1} \\
& \quad-\frac{y^{1}-y^{2}+y^{3}-y^{4}+\cdots+y^{r-1}-y^{r}}{r-1} \mid \\
& \leq \frac{r}{r+1} \frac{1}{r}\left\{\frac{\left|x^{1}-y^{1}\right|+\left|x^{2}-y^{2}\right|+\cdots+\left|x^{r}-y^{r}\right|}{r-1}\right\} \\
& =\frac{r}{r+1} \frac{1}{r}\left(d\left(g x^{1}, g y^{1}\right)+d\left(g x^{2}, g y^{2}\right)\right. \\
& \left.\quad+\cdots+d\left(g x^{r}, g y^{r}\right)\right) \\
& =\phi\left(\frac{1}{r} \sum_{n=1}^{r} d\left(g x^{n}, g y^{n}\right)\right) .
\end{aligned}
$$

Thus all the conditions of Theorem 13 (without order) are satisfied and $(0,0, \ldots, 0)$ is a $r$-tupled coincidence point of $F$ and $g$.

\section{Acknowledgments}

All the authors are grateful to both the learned referees for their fruitful suggestions and remarks towards the improvement of this paper.

\section{References}

[1] D. W. Boyd and J. S. W. Wong, "On nonlinear contractions," Proceedings of the American Mathematical Society, vol. 20, pp. 458-464, 1969.

[2] Lj. B. Cirić, "A generalization of Banach's contraction principle," Proceedings of the American Mathematical Society, vol. 45, pp. 267-273, 1974.

[3] Lj. B. Cirić and J. S. Ume, "Multi-valued non-self-mappings on convex metric spaces," Nonlinear Analysis: Theory, Methods \& Applications A, vol. 60, no. 6, pp. 1053-1063, 2005.

[4] Lj. B. Cirić, "Coincidence and fixed points for maps on topological spaces," Topology and Its Applications, vol. 154, no. 17, pp. 3100-3106, 2007.

[5] Lj. B. Cirić, "Fixed point theorems for multi-valued contractions in complete metric spaces," Journal of Mathematical Analysis and Applications, vol. 348, no. 1, pp. 499-507, 2008.

[6] D. Dorić, Z. Kadelburg, and S. Radenović, "Coupled fixed point results for mappings without mixed monotone property," Applied Mathematics Letters, vol. 25, no. 11, pp. 1803-1808, 2012.

[7] N. Hussain, "Common fixed points in best approximation for Banach operator pairs with Cirić type I-contractions," Journal of Mathematical Analysis and Applications, vol. 338, no. 2, pp. 1351-1363, 2008.

[8] M. Jleli, V. Ćojbašić-Rajić, B. Samet, and C. Vetro, "Fixed point theorems on ordered metric spaces and applications to Nonlinear beam equations," Journal of Fixed Point Theory and Its Applications. In press.

[9] Z. Liu, Z. Guo, S. M. Kang, and S. K. Lee, "On Cirić type mappings with nonunique fixed and periodic points," International Journal of Pure and Applied Mathematics, vol. 26, no. 3, pp. 399408, 2006.
[10] J. J. Nieto and R. Rodríguez-López, “Contractive mapping theorems in partially ordered sets and applications to ordinary differential equations," Order, vol. 22, no. 3, pp. 223-239, 2005.

[11] J. J. Nieto and R. Rodríguez-López, "Existence and uniqueness of fixed point in partially ordered sets and applications to ordinary differential equations," Acta Mathematica Sinica (English Series), vol. 23, no. 12, pp. 2205-2212, 2007.

[12] H. K. Pathak, Y. J. Cho, and S. M. Kang, "An application of fixed point theorems in best approximation theory," International Journal of Mathematics and Mathematical Sciences, vol. 21, no. 3, pp. 467-470, 1998.

[13] A. C. M. Ran and M. C. B. Reurings, "A fixed point theorem in partially ordered sets and some applications to matrix equations," Proceedings of the American Mathematical Society, vol. 132, no. 5, pp. 1435-1443, 2004.

[14] B. K. Ray, "On Ciric's fixed point theorem," Fundamenta Mathematicae, vol. 94, no. 3, pp. 221-229, 1977.

[15] H. Aydi, "Some coupled fixed point results on partial metric spaces," International Journal of Mathematics and Mathematical Sciences, vol. 2011, Article ID 647091, 11 pages, 2011.

[16] B. S. Choudhury and A. Kundu, "A coupled coincidence point result in partially ordered metric spaces for compatible mappings," Nonlinear Analysis: Theory, Methods \& Applications A, vol. 73, no. 8, pp. 2524-2531, 2010.

[17] B. S. Choudhury and P. Maity, "Coupled fixed point results in generalized metric spaces," Mathematical and Computer Modelling, vol. 54, no. 1-2, pp. 73-79, 2011.

[18] B. S. Choudhury, N. Metiya, and A. Kundu, "Coupled coincidence point theorems in ordered metric spaces," Annali dell'Universitá di Ferrara, vol. 57, no. 1, pp. 1-16, 2011.

[19] F. Sabetghadam, H. P. Masiha, and A. H. Sanatpour, "Some coupled fixed point theorems in cone metric spaces," Fixed Point Theory and Applications, vol. 2009, Article ID 125426, 8 pages, 2009.

[20] H. Aydi, "Some coupled fixed point results on partial metric spaces," International Journal of Mathematics and Mathematical Sciences, vol. 2011, Article ID 647091, 11 pages, 2011.

[21] V. Lakshmikantham and L. Cirić, "Coupled fixed point theorems for nonlinear contractions in partially ordered metric spaces," Nonlinear Analysis: Theory, Methods \& Applications A, vol. 70, no. 12, pp. 4341-4349, 2009.

[22] T. Gnana Bhaskar and V. Lakshmikantham, "Fixed point theorems in partially ordered metric spaces and applications," Nonlinear Analysis: Theory, Methods \& Applications A, vol. 65, no. 7, pp. 1379-1393, 2006. 


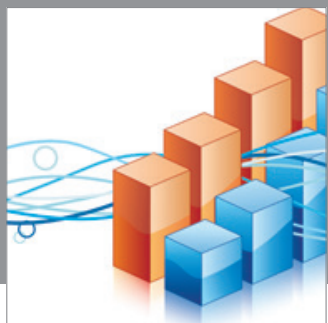

Advances in

Operations Research

mansans

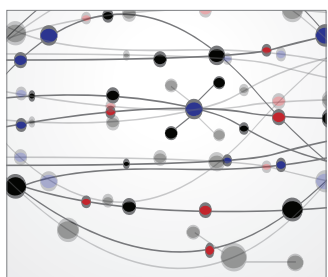

The Scientific World Journal
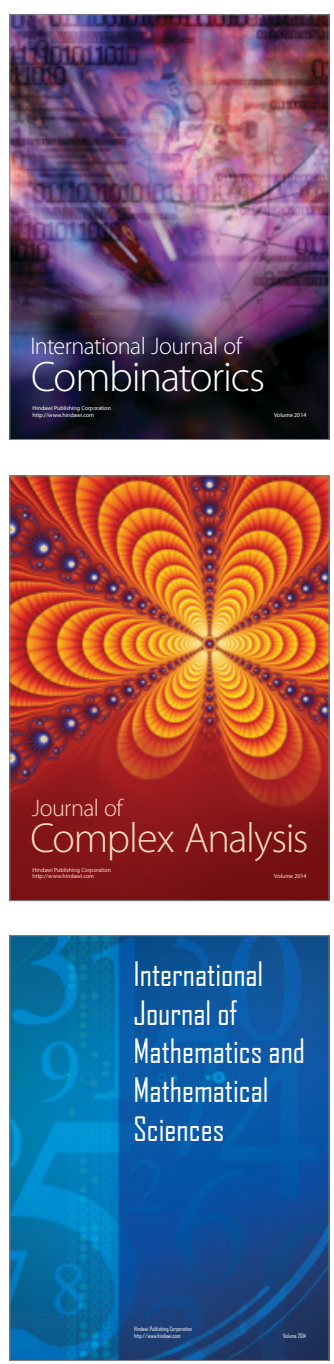
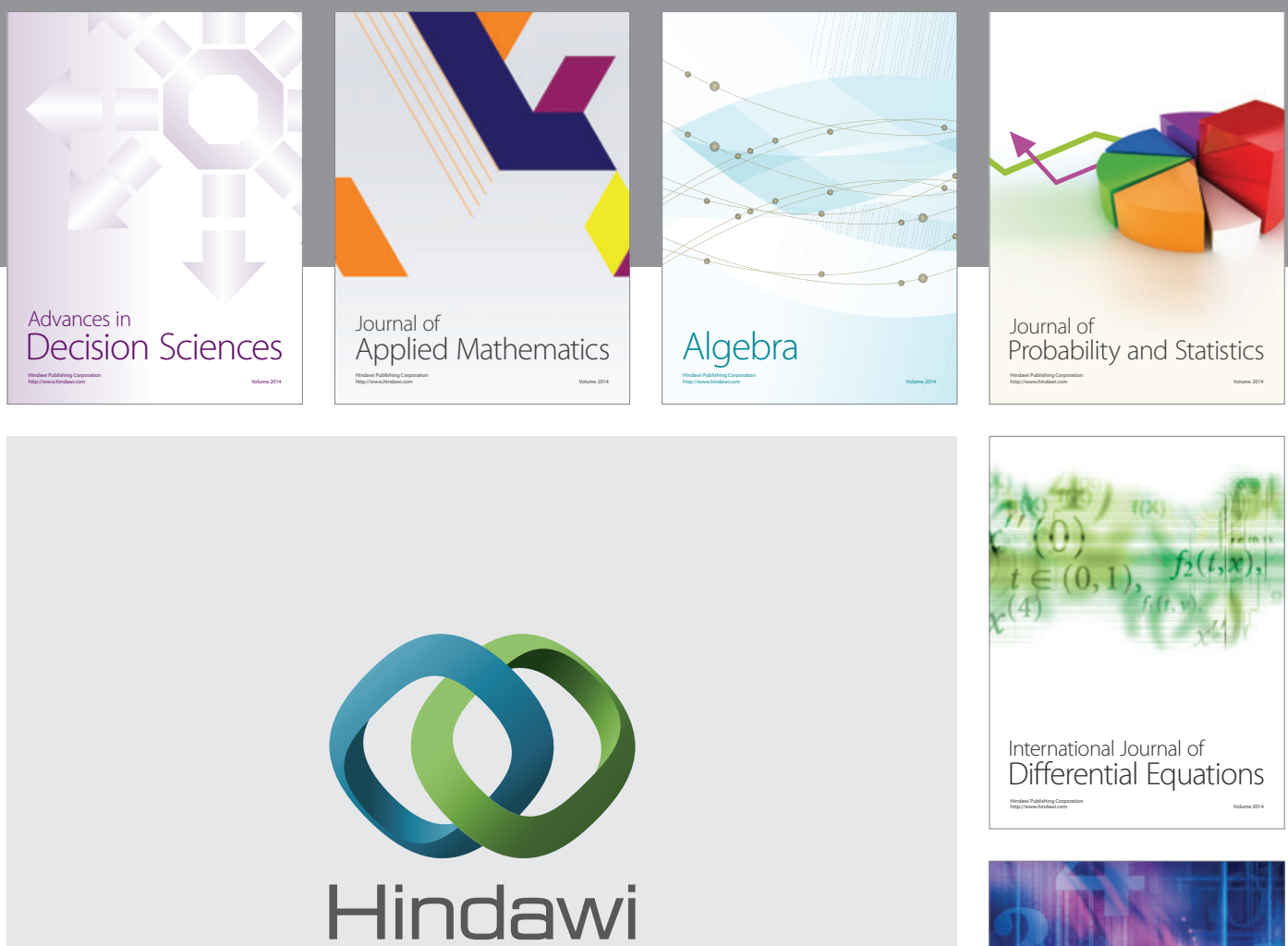

Submit your manuscripts at http://www.hindawi.com
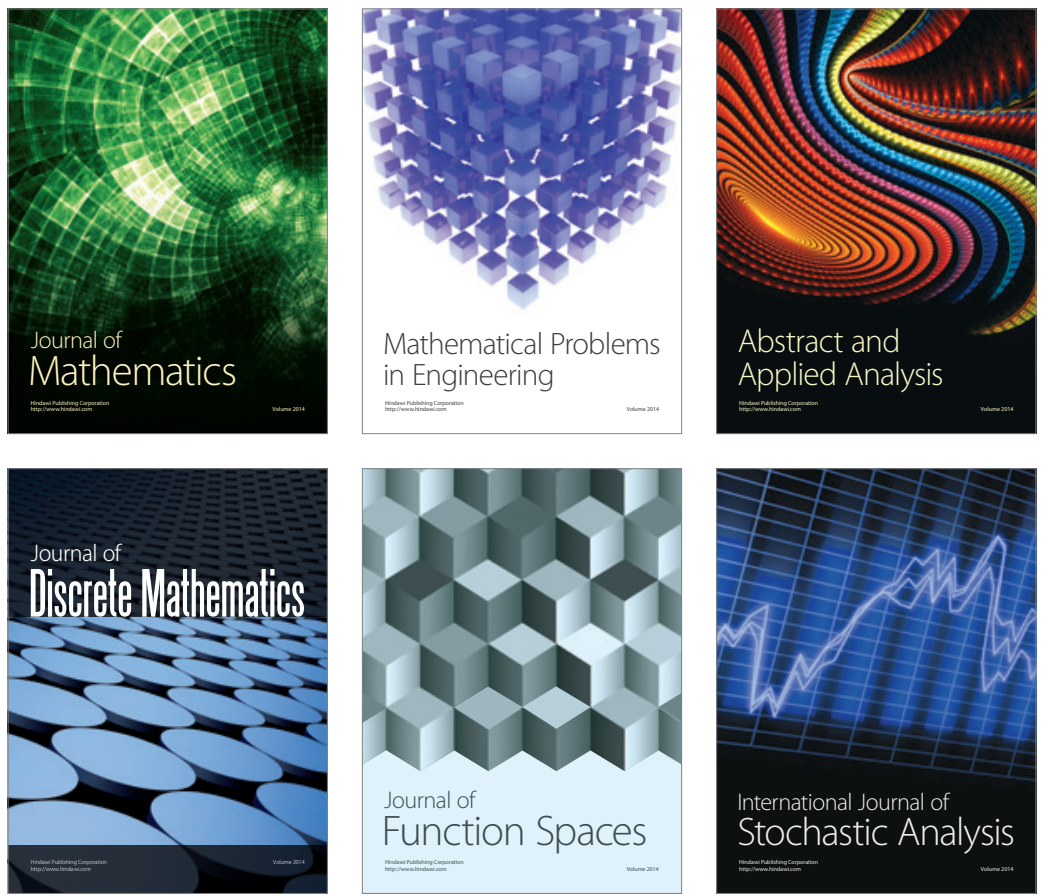

Journal of

Function Spaces

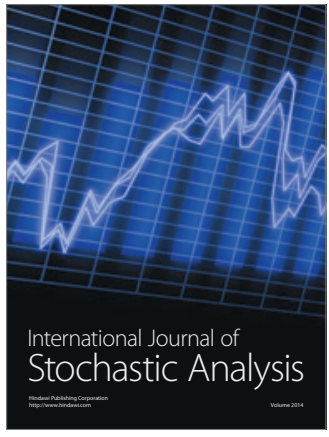

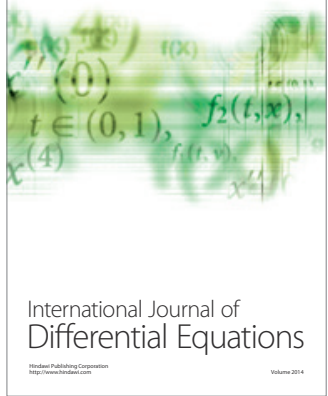
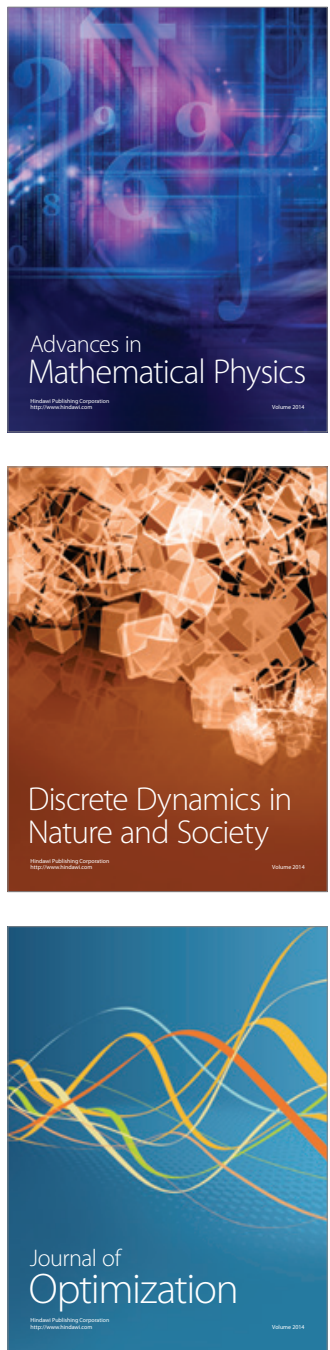